\title{
Associated consistency and values for TU games
}

\author{
Theo S. H. Driessen
}

Accepted: 21 December 2009 / Published online: 6 February 2010

(C) The Author(s) 2010. This article is published with open access at Springerlink.com

\begin{abstract}
In the framework of the solution theory for cooperative transferable utility games, Hamiache axiomatized the well-known Shapley value as the unique one-point solution verifying the inessential game property, continuity, and associated consistency. The purpose of this paper is to extend Hamiache's axiomatization to the class of efficient, symmetric, and linear values, of which the Shapley value is the most important representative. For this enlarged class of values, explicit relationships to the Shapley value are exploited in order to axiomatize such values with reference to a slightly adapted inessential game property, continuity, and a similar associated consistency. The latter axiom requires that the solutions of the initial game and its associated game (with the same player set, but a different characteristic function) coincide.
\end{abstract}

Keywords Cooperative game $\cdot$ Associated game $\cdot$ Linear value . Shapley value $\cdot$ Consistency

JEL Classification C71

\section{Introduction}

Formally, a transferable utility game (or cooperative game or coalitional game with side payments) is a pair $\langle N, v\rangle$, where $N$ is a finite set of at least two players and $v: 2^{N} \rightarrow \mathbb{R}$ is a characteristic function satisfying $v(\emptyset)=0$. An element of $N$

Revised version submitted May 1, 2006 (referee reports dated May 17, 2005).

T. S. H. Driessen ( $\varangle)$

Department of Applied Mathematics, Faculty of Electrical Engineering, Mathematics and Computer Science, University of Twente, P.O. Box 217, 7500 AE, Enschede, The Netherlands

e-mail: t.s.h.driessen@ewi.utwente.nl 
(notation: $i \in N$ ) and a nonempty subset $S$ of $N$ (notation: $S \subseteq N$ or $S \in 2^{N}$ with $S \neq \emptyset$ ) is called a player and coalition respectively, and the real number $v(S)$ is called the worth of coalition $S$. The size (cardinality) of coalition $S$ is denoted by $|S|$ or, if no ambiguity is possible, by $s$. Particularly, $n$ denotes the size of the player set $N$. Let $\mathcal{G}$ denote the universal game space consisting of all TU games. A TU game $\langle N, v\rangle$ is called superadditive if $v(S \cup T) \geq v(S)+v(T)$ for all $S, T \subseteq N$ with $S \cap T=\emptyset$.

The solution part of cooperative game theory deals with the allocation problem of how to divide, for any TU game $\langle N, v\rangle$, the worth $v(N)$ of the grand coalition $N$ among the players. Particularly, the one-point solution theory associates with any TU game a single allocation called the value of the TU game. Formally, a value on $\mathcal{G}$ is a function $\Phi$ that assigns a single payoff vector $\Phi(N, v)=\left(\Phi_{i}(N, v)\right)_{i \in N} \in \mathbb{R}^{N}$ to every game $\langle N, v\rangle \in \mathcal{G}$. The so-called value $\Phi_{i}(N, v)$ of player $i$ in the game $\langle N, v\rangle$ represents an assessment by $i$ of his gains for participating in the game. Without going into details, the well-known Shapley value $\operatorname{Sh}(N, v)=\left(\operatorname{Sh}_{i}(N, v)\right)_{i \in N} \in \mathbb{R}^{N}$ is as follows (Driessen 1988; Roth 1988; Shapley 1953): for all $i \in N$

$$
S h_{i}(N, v)=\sum_{S \subseteq N \backslash\{i\}} \frac{1}{n \cdot\left(\begin{array}{c}
n-1 \\
s
\end{array}\right)} \cdot[v(S \cup\{i\})-v(S)] .
$$

The eldest axiomatization of the Shapley value is stated in 1953 by Shapley himself by referring to four properties called efficiency, symmetry, linearity, and dummy player property. The most recent axiomatization of the Shapley value is stated by Hamiache (2001) who proposed a new set of axioms called the "inessential game property", "continuity", and "associated consistency". The purpose of this paper is to extend Hamiache's axiomatization to the class of efficient, symmetric, and linear values, of which the Shapley value is the most important representative. For this enlarged class of values, explicit relationships to the Shapley value will be exploited to provide an axiomatization based on a slightly adapted inessential game property, continuity, and a similar associated consistency. Concerning values for games, firstly we review several properties treated in former axiomatizations of the Shapley value and secondly, we recall four equivalent interpretations of the class of efficient, symmetric, and linear values.

Definition 1 A value $\Phi$ on the universal game space $\mathcal{G}$ possesses

(i) efficiency, if $\sum_{i \in N} \Phi_{i}(N, v)=v(N)$ for all games $\langle N, v\rangle$;

(ii) symmetry, if $\Phi_{\pi(i)}(N, \pi v)=\Phi_{i}(N, v)$ for all games $\langle N, v\rangle$, all $i \in N$, and every permutation $\pi$ on $N$. Here the game $\langle N, \pi v\rangle$ is defined by $(\pi v)(\pi S):=v(S)$ for all $S \subseteq N$;

(iii) linearity, if $\Phi(N, \alpha \cdot v+\beta \cdot w)=\alpha \cdot \Phi(N, v)+\beta \cdot \Phi(N, w)$ for all games $\langle N, v\rangle,\langle N, w\rangle$, and all $\alpha \in \mathbb{R}, \beta \in \mathbb{R}$. Here the game $\langle N, \alpha \cdot v+\beta \cdot w\rangle$ is defined by $(\alpha \cdot v+\beta \cdot w)(S):=\alpha \cdot v(S)+\beta \cdot w(S)$ for all $S \subseteq N$;

(iv) inessential game property, if $\Phi_{i}(N, v)=v(\{i\})$ for all inessential games $\langle N, v\rangle$, and all $i \in N$. Here the game $\langle N, v\rangle$ is inessential if $v(S)=\sum_{j \in S} v(\{j\})$ for all $S \subseteq N, S \neq \emptyset$; 
(v) continuity, if for any (pointwise) convergent sequence $\left\{\left\langle N, v_{m}\right\rangle\right\}_{m=0}^{\infty}$ of games, say the limit of which is the game $\langle N, \tilde{v}\rangle$, the corresponding sequence $\left\{\Phi\left(N, v_{m}\right)\right\}_{m=0}^{\infty}$ of values converges to the value $\Phi(N, \tilde{v})$.

Throughout this paper we deal with two adaptations of games. The first adaptation of a given game $\langle N, v\rangle$ concerns the following optimistic self-evaluation by coalitions. Every coalition $S$ evaluates its own worth $v_{\lambda}^{S h}(S)$ in the associated game $\left\langle N, v_{\lambda}^{S h}\right\rangle$ as the sum of its initial worth $v(S)$ and a percentage $\lambda \in[0,1]$ of all possible surpluses $v(S \cup\{j\})-v(S)-v(\{j\})$ arising from mutual cooperation (instead of non-cooperation) among $S$ itself and $j \in N \backslash S$. ${ }^{1}$ For superadditive games $\langle N, v\rangle$, we have $v_{\lambda}^{S h}(S) \geq v(S)$ for all $S \subseteq N$, while for inessential games $\langle N, v\rangle v_{\lambda}^{S h}(S)=v(S)$. Further, the worth of the grand coalition $N$ does not change under this adaptation, i.e., $v_{\lambda}^{S h}(N)=v(N)$.

Associated consistency requires that the value be invariant under the adaptation of the game into its associated game. Because payoffs to players neither increase nor decrease, there are not adverse effects of such optimistic self-evaluations by coalitions.

Definition 2 (cf. Hamiache 2001)

(i) Given any game $\langle N, v\rangle$ and $\lambda \in[0,1]$, define its (standard) associated game $\left\langle N, v_{\lambda}^{S h}\right\rangle$ as follows: for all $S \subseteq N$,

$$
v_{\lambda}^{S h}(S):=v(S)+\lambda \cdot \sum_{j \in N \backslash S}[v(S \cup\{j\})-v(S)-v(\{j\})] .
$$

(ii) A value $\Phi$ on $\mathcal{G}$ possesses associated consistency with respect to the associated game of (2) if $\Phi\left(N, v_{\lambda}^{S h}\right)=\Phi(N, v)$ for all games $\langle N, v\rangle$, and all $\lambda \in[0,1]$.

The second adaptation of a game concerns a check of credibility of the characteristic function by an independent arbiter. The task of the arbiter is to perform a scaling operation by taking into account the sizes of both the player set and the coalition (but not the coalition itself). That is, for every coalition $S \subseteq N$, its initial worth $v(S)$ is scaled down or up to $b_{s}^{n} \cdot v(S)$ by some non-negative scaling number $b_{s}^{n} \geq 0$, where $s$ denotes the size of coalition $S$. For example, if $b_{s}^{n}=\frac{1}{s}$, then the scaling operation involves averaging the worth of any coalition different from the grand coalition $N$. By convention, $b_{n}^{n}=1$ in order to guarantee the invariant worth of the grand coalition. Throughout the remainder of the paper, let $\mathbb{N}=\{0,1,2,3, \ldots\}$ denote the set of all natural numbers and let $\mathcal{B}=\left\{b_{s}^{n} \mid n \in \mathbb{N} \backslash\{0,1\}, s=1,2, \ldots, n\right\}$ denote a collection of non-negative scaling constants with $b_{n}^{n}=1$ for all $n \geq 2$. No scaling at all is expressed by the collection $\mathcal{U}$ of unit constants (equal to one).

\footnotetext{
1 To be coherent with Hamiache's (2001, pp. 281-282) myopic vision of the environment, every coalition $S$ ignores the links existing between players in $N \backslash S$. As a consequence, a coalition $S$ considers itself at the center of a star-like graph, which is equivalent to say that coalition $S$ considers players in $N \backslash S$ as isolated elements. Following the additional "divide and rule" behavior of the coalitions, $S$ may believe that the appropriation of at least a part of the surpluses $v(S \cup\{j\})-v(S)-v(\{j\})$, generated by its cooperation with each one of the isolated players $j \in N \backslash S$, is within reach.
} 
Definition 3 Given a collection $\mathcal{B}$ of non-negative scaling constants and any game $\langle N, v\rangle$ with at least two players, define its $\mathcal{B}$-scaled game $\langle N, \mathcal{B} v\rangle$ as follows: $(\mathcal{B} v)(\emptyset):=0$ and

$$
(\mathcal{B} v)(S):=b_{s}^{n} \cdot v(S) \text { for all } S \subseteq N, S \neq \emptyset .
$$

In view of the explicit formula (1), it follows immediately that the Shapley value possesses the inessential game property and continuity, whereas non-trivial calculations are necessary to verify the associated consistency of the Shapley value with respect to the associated game of (2), that is $\operatorname{Sh}\left(N, v_{\lambda}^{S h}\right)=\operatorname{Sh}(N, v)$ for all games $\langle N, v\rangle$, and all $\lambda \in[0,1]$. The main theorem in Hamiache (2001, p. 282) states that the Shapley value is the unique value verifying the inessential game property, continuity, and associated consistency with respect to the associated game of (2) (provided $0<\lambda<\frac{2}{n}$ ). The uniqueness proof is rather tough and full of combinatorial calculations.

In the remainder, we aim to develop a similar axiomatization for any efficient, symmetric, and linear value. The explicit relationship between the Shapley value and any efficient, symmetric, and linear value is listed in the following fundamental theorem. One out of four equivalent statements refers to the adaptation of the game into its $\mathcal{B}$-scaled game.

Theorem 1 (Equivalence Theorem) The next four statements for a value $\psi$ on the universal game space $\mathcal{G}$ are equivalent.

(i) $\psi$ possesses efficiency, symmetry, and linearity.

(ii) There exists a (unique) collection of constants $\left\{\rho_{s}^{n} \mid n \in \mathbb{N} \backslash\{0,1\}, s=\right.$ $1,2, \ldots, n-1\}$ such that, for every n-person game $\langle N, v\rangle$ with at least two players, the value payoff vector $\left(\psi_{i}(N, v)\right)_{i \in N} \in \mathbb{R}^{N}$ is of the following form (Ruiz 1998, Lemma 9, p. 117): for all $i \in N$

$$
\psi_{i}(N, v)=\frac{v(N)}{n}+\sum_{\substack { S \subsetneq N \\
\begin{subarray}{c}{\ddagger \\
S \ni i{ S \subsetneq N \\
\begin{subarray} { c } { \ddagger \\
S \ni i } }\end{subarray}} \frac{\rho_{s}^{n}}{s} \cdot v(S)-\sum_{\substack{S \subseteq N, S \not \supset i}} \frac{\rho_{s}^{n}}{n-s} \cdot v(S) .
$$

(iii) There exists a (unique) collection of constants $\mathcal{B}=\left\{b_{s}^{n} \mid n \in \mathbb{N} \backslash\{0,1\}, s=\right.$ $1,2, \ldots, n\}$ with $b_{n}^{n}:=1$ such that, for every n-person game $\langle N, v\rangle$ with at least two players, the value payoff vector $\left(\psi_{i}(N, v)\right)_{i \in N} \in \mathbb{R}^{N}$ is of the following form: for all $i \in N$

$$
\psi_{i}(N, v)=\sum_{S \subseteq N \backslash\{i\}} \frac{1}{n \cdot\left(\begin{array}{c}
n-1 \\
s
\end{array}\right)} \cdot\left[b_{s+1}^{n} \cdot v(S \cup\{i\})-b_{s}^{n} \cdot v(S)\right] .
$$

(iv) There exists a (unique) collection of constants $\mathcal{B}=\left\{b_{s}^{n} \mid n \in \mathbb{N} \backslash\{0,1\}, s=\right.$ $1,2, \ldots, n\}$ with $b_{n}^{n}:=1$ such that $\psi(N, v)=\operatorname{Sh}(N, \mathcal{B} v)$ for every game $\langle N, v\rangle$ with at least two players, i.e.,

$$
\psi_{i}(N, v)=S h_{i}(N, \mathcal{B} v) \quad \text { for all } i \in N
$$


It is left to the reader to verify, by straightforward computations, that the expression on the right hand of (3) agrees with the one on the right hand of (4) by choosing $b_{s}^{n}=\left(\begin{array}{l}n \\ s\end{array}\right) \cdot \rho_{s}^{n}$ for all $s \in\{1,2, \ldots, n-1\}$. Whenever $b_{s}^{n}=1$ for all $s \in\{1,2, \ldots, n\}$, the expression on the right hand of (4) reduces to the Shapley value payoff (1) of player $i$ in the $n$-person game $\langle N, v\rangle$ itself, that is, $\psi=S h$. Generally speaking, in view of (1), the right hand of (4) equals the Shapley value payoff $S h_{i}(N, \mathcal{B} v)$ of player $i$ in the $\mathcal{B}$-scaled game $\langle N, \mathcal{B} v\rangle$. In summary, the Equivalence Theorem 1 states that a value $\psi$ is efficient, symmetric, and linear if and only if the $\psi$-value of a game coincides with the Shapley value of the $\mathcal{B}$-scaled game. Throughout the development of the new theory and the forthcoming proofs we prefer to use the equality $\psi(N, v)=\operatorname{Sh}(N, \mathcal{B} v)$ among value payoff vectors.

Example 1 Given the separable contribution $S C_{i}(N, v):=v(N)-v(N \backslash\{i\})$ of any player $i \in N$ in the game $\langle N, v\rangle$, the egalitarian distribution of the remaining nonseparable contribution $N S C(N, v):=v(N)-\sum_{j \in N} S C_{j}(N, v)$ yields the so-called egalitarian non-separable contribution ENSC-value. That is, for all games $\langle N, v\rangle$,

$$
\operatorname{ENSC}_{i}(N, v)=S C_{i}(N, v)+\frac{N S C(N, v)}{n} \text { for all } i \in N
$$

It is easy to verify that the $E N S C$-value is an efficient, symmetric, and linear value and in fact, its corresponding collection $\mathcal{B}=\left\{b_{s}^{n}\right\}$ of scaling constants is given by $b_{n}^{n}=1, b_{n-1}^{n}=n-1$, and $b_{s}^{n}=0$ for all $1 \leq s \leq n-2$. By Equivalence Theorem $1, \operatorname{ENSC}(N, v)=\operatorname{Sh}(N, \mathcal{B} v)$ for all games $\langle N, v\rangle$.

Definition 4 Let $\mathcal{B}=\left\{b_{s}^{n}\right\}$ be a collection of non-negative scaling constants with $b_{n}^{n}=1$ for all $n \geq 2$.

(i) A game $\langle N, v\rangle$ is called $\mathcal{B}$-inessential if its $\mathcal{B}$-scaled game $\langle N, \mathcal{B} v\rangle$ is inessential, i.e., $(\mathcal{B} v)(S)=\sum_{j \in S}(\mathcal{B} v)(\{j\})$ for all $S \subseteq N, S \neq \emptyset$, or equivalently, $b_{s}^{n} \cdot v(S)=\sum_{j \in S} b_{1}^{n} \cdot v(\{j\})$.

(ii) A value $\Phi$ on $\mathcal{G}$ possesses $\mathcal{B}$-inessential game property if $\Phi_{i}(N, v)=b_{1}^{n} \cdot v(\{i\})$ for all $\mathcal{B}$ - inessential games $\langle N, v\rangle$, and all $i \in N$.

Alternatively, with respect to a given collection of positive constants $\mathcal{B}$, a game $\langle N, v\rangle$ is $\mathcal{B}$-inessential if and only if there exists a vector $\mathbf{x}=\left(x_{k}\right)_{k \in N} \in \mathbb{R}^{N}$ such that $v(S)=\frac{b_{1}^{n}}{b_{s}^{n}} \cdot \sum_{j \in S} x_{j}$ for all $S \subseteq N, S \neq \emptyset$. In words, a game is $\mathcal{B}$-inessential if it is decomposable as a product of an inessential game (associated with some vector $\left.\mathbf{x} \in \mathbb{R}^{N}\right)$ and a $\mathcal{B}$-symmetric game.

For example, the game $\langle N, v\rangle$ with characteristic function $v(S)=|S|^{2}$ for all $S \varsubsetneqq N$, and $v(N)=|N|$, is $\mathcal{B}$-inessential with respect to the averaging collection of constants $b_{s}^{n}=\frac{1}{s}$. Generally speaking, for arbitrary positive numbers $\alpha$ and $p$, the game $\langle N, v\rangle$ with characteristic function $v(S)=\alpha \cdot|S|^{p}$ for all $S \varsubsetneqq N$, and $v(N)=\alpha \cdot|N|$, is $\mathcal{B}$-inessential with respect to the collection of constants $b_{s}^{n}=s^{1-p}$.

We conclude this section with the claim that any efficient, symmetric, and linear value $\psi$ on $\mathcal{G}$ satisfies the $\mathcal{B}$-inessential game property, provided the collection of non-negative constants $\mathcal{B}$ corresponds to $\psi$ through (5). Indeed, by applying the 
equivalence (5) and the well-known inessential game property for the Shapley value to the $\mathcal{B}$-scaled game, it follows that $\psi_{i}(N, v)=S h_{i}(N, \mathcal{B} v)=(\mathcal{B} v)(\{i\})=b_{1}^{n} \cdot v(\{i\})$ for all $\mathcal{B}$-inessential games $\langle N, v\rangle$, and all $i \in N$. For example, the $E N S C$-value satisfies the $\mathcal{B}$-inessential game property, where the scaling constants are zero, except for $b_{n}^{n}=1, b_{n-1}^{n}=n-1$.

\section{Extended associated consistency}

Recall that an independent arbiter is supposed to scale down or up any game through a collection of non-negative scaling constants $\mathcal{B}$. Incorporating this scaling operation, the $\mathcal{B}$-associated game is meant to represent the optimistic self-evaluation by coalitions, mathematically expressed as the associated game of the scaled version of the initial game. Throughout this section let $\mathcal{B}=\left\{b_{s}^{n}\right\}$ be a collection of positive scaling constants with $b_{n}^{n}=1$ for all $n \geq 2$.

Definition 5 Let $\mathcal{U}$ denote the collection of unit constants (equal to one).

(i) Given any game $\langle N, v\rangle$ and $\lambda \in[0,1]$, define its $\mathcal{B}$-associated game $\left\langle N, v_{\lambda}^{\mathcal{B}}\right\rangle$ as follows: $v_{\lambda}^{\mathcal{B}}(\emptyset):=0$ and for all $S \subseteq N, S \neq \emptyset$,

$$
\left(\mathcal{B}\left(v_{\lambda}^{\mathcal{B}}\right)\right)(S):=(\mathcal{B} v)(S)+\lambda \sum_{j \in N \backslash S}[(\mathcal{B} v)(S \cup\{j\})-(\mathcal{B} v)(S)-(\mathcal{B} v)(\{j\})],
$$

or equivalently,

$$
b_{s}^{n} v_{\lambda}^{\mathcal{B}}(S):=b_{s}^{n} v(S)+\lambda \sum_{j \in N \backslash S}\left[b_{s+1}^{n} v(S \cup\{j\})-b_{s}^{n} v(S)-b_{1}^{n} v(\{j\})\right] .
$$

(ii) Particularly, for all games $\langle N, w\rangle$, all $\lambda \in[0,1]$, and all $S \subseteq N, S \neq \emptyset$,

$$
w_{\lambda}^{\mathcal{U}}(S):=w(S)+\lambda \cdot \sum_{j \in N \backslash S}[w(S \cup\{j\})-w(S)-w(\{j\})] .
$$

(iii) A value $\Phi$ on $\mathcal{G}$ satisfies $\mathcal{B}$-consistency with respect to the $\mathcal{B}$-associated game of (6) if $\Phi\left(N, v_{\lambda}^{\mathcal{B}}\right)=\Phi(N, v)$ for all games $\langle N, v\rangle$, and all $\lambda \in[0,1]$.

Firstly, note that the $\mathcal{U}$-associated game agrees with the standard associated game, i.e., $\left\langle N, v_{\lambda}^{\mathcal{U}}\right\rangle=\left\langle N, v_{\lambda}^{S h}\right\rangle$. Secondly, notice that the $\mathcal{B}$-scaled version of the $\mathcal{B}$-associated game $\left\langle N, v_{\lambda}^{\mathcal{B}}\right\rangle$ agrees with the $\mathcal{U}$-associated game of the $\mathcal{B}$-scaled version of the initial game $\langle N, v\rangle$. In formula, for all games $\langle N, v\rangle$, and all $\lambda \in[0,1]$,

$$
\left\langle N, \mathcal{B}\left(v_{\lambda}^{\mathcal{B}}\right)\right\rangle=\left\langle N,(\mathcal{B} v)_{\lambda}^{\mathcal{U}}\right\rangle
$$

Alternatively, it turns out that the $\mathcal{B}$-scaling operator and the two associated game operators with respect to the two collections of scaling constants $\mathcal{B}$ and $\mathcal{U}$ generate a commutative diagram. The scaling operation requires a collection of positive scaling 
constants $\mathcal{B}$ since zero constants may cause problems for the $\mathcal{B}$-associated game to be well-defined. For example, in the setting of the ENSC-value, its corresponding collection of scaling constants $\mathcal{B}=\left\{b_{s}^{n}\right\}$ is given by $b_{n}^{n}=1, b_{n-1}^{n}=n-1$, and $b_{s}^{n}=0$ for all $1 \leq s \leq n-2$. Consequently, (6) is not solvable for $v_{\lambda}^{\mathcal{B}}(S)$ with $|S|=n-2$ due to $b_{n-2}^{n}=0, b_{1}^{n}=0$, whereas $b_{n-1}^{n} \neq 0$.

Proposition 1 Let $\psi$ be the efficient, symmetric, and linear value on $\mathcal{G}$, corresponding to a collection of positive scaling constants $\mathcal{B}$. Then

(i) $\psi$ satisfies $\mathcal{B}$-consistency, i.e., $\psi\left(N, v_{\lambda}^{\mathcal{B}}\right)=\psi(N, v)$ for all games $\langle N, v\rangle$, and all $\lambda \in[0,1]$.

(ii) $\psi$ satisfies the $\mathcal{B}$-inessential game property.

(iii) $\psi$ satisfies continuity.

Proof (i) By applying the Equivalence Theorem to the value $\psi$ twice, the game equality (7), and the $\mathcal{U}$-consistency for the Shapley value (Hamiache 2001, p. 282) applied to the $\mathcal{B}$-scaled game, respectively, it follows for all games $\langle N, v\rangle \in \mathcal{G}$, and all $\lambda \in[0,1]$,

$$
\psi\left(N, v_{\lambda}^{\mathcal{B}}\right) \stackrel{(5)}{=} \operatorname{Sh}\left(N, \mathcal{B}\left(v_{\lambda}^{\mathcal{B}}\right)\right) \stackrel{(7)}{=} \operatorname{Sh}\left(N,(\mathcal{B} v)_{\lambda}^{\mathcal{U}}\right)=\operatorname{Sh}(N, \mathcal{B} v) \stackrel{(5)}{=} \psi(N, v) .
$$

This proves $\mathcal{B}$-consistency for $\psi$, whereas the $\mathcal{B}$-inessential game property for $\psi$ was already proven at the end of the previous section.

(iii) Finally, continuity for $\psi$ holds because the Shapley value satisfies continuity. Given any (pointwise) convergent sequence $\left\{\left\langle N, v_{k}\right\rangle\right\}_{k=0}^{\infty}$ of games, say the limit of which is the game $\langle N, \tilde{v}\rangle$, the sequence $\left\{\left\langle N, \mathcal{B}\left(v_{k}\right)\right\rangle\right\}_{k=0}^{\infty}$ of $\mathcal{B}$-scaled games converges too, the limit of which is the $\mathcal{B}$-scaled game $\langle N, \mathcal{B} \tilde{v}\rangle$. Consequently, the corresponding sequence $\left\{\operatorname{Sh}\left(N, \mathcal{B}\left(v_{k}\right)\right)\right\}_{k=0}^{\infty}$ of Shapley values converges to the Shapley value $\operatorname{Sh}(N, \mathcal{B} \tilde{v})$, or equivalently, the corresponding sequence $\left\{\psi\left(N, v_{k}\right)\right\}_{k=0}^{\infty}$ of values converges to the value $\psi(N, \tilde{v})$.

Now we are able to state our main result, an axiomatic characterization of any efficient, symmetric, and linear value. The uniqueness part of its proof will be treated in the next section.

Theorem 2 Let $\mathcal{B}$ be a collection of positive scaling constants. There exists a unique value $\Phi$ on $\mathcal{G}$ verifying the $\mathcal{B}$-inessential game property, continuity, and $\mathcal{B}$-consistency with respect to the $\mathcal{B}$-associated game of $(6)$ (provided $0<\lambda<\frac{2}{n}$ ). The value $\Phi$ is the efficient, symmetric, and linear value $\psi$ on $\mathcal{G}$ induced by $\mathcal{B}$ (see (4) or equivalently, $\psi(N, v)=\operatorname{Sh}(N, \mathcal{B} v)$ for all games $\langle N, v\rangle)$.

\section{Proof of uniqueness part of Theorem 2}

Throughout this section, let $\mathcal{B}=\left\{b_{s}^{n}\right\}$ be a collection of positive scaling constants with $b_{n}^{n}=1$ for all $n \geq 2$. In comparison to Hamiache's proof of uniqueness in the setting of the Shapley value, our current approach is similar by applying the adapted $\mathcal{B}$-associated game procedure repeatedly. That is, the notion of $m$-repeated $\mathcal{B}$-associated game $\left\langle N, v_{\lambda}^{m * \mathcal{B}}\right\rangle$ will be introduced in such a way that $v_{\lambda}^{1 * \mathcal{B}}=v_{\lambda}^{\mathcal{B}}$ and $v_{\lambda}^{2 * \mathcal{B}}=\left(v_{\lambda}^{\mathcal{B}}\right)_{\lambda}^{\mathcal{B}}$ and $v_{\lambda}^{3 * \mathcal{B}}=\left(v_{\lambda}^{2 * \mathcal{B}}\right)_{\lambda}^{\mathcal{B}}$ and so on. 
Definition 6 Let $\mathcal{B}$ be a collection of positive scaling constants, $\langle N, v\rangle \in \mathcal{G}$, and $\lambda \in[0,1]$. Recall Definition 5 of the $\mathcal{B}$-associated game $\left\langle N, v_{\lambda}^{\mathcal{B}}\right\rangle$ of $(6)$.

(i) For all $m \in \mathbb{N}$, let the $m$-repeated $\mathcal{B}$-associated game $\left\langle N, v_{\lambda}^{m * \mathcal{B}}\right\rangle$ be defined recursively by $v_{\lambda}^{m * \mathcal{B}}:=\left(v_{\lambda}^{(m-1) * \mathcal{B}}\right)_{\lambda}^{\mathcal{B}}$, where $v_{\lambda}^{0 * \mathcal{B}}:=v$ and $v_{\lambda}^{1 * \mathcal{B}}:=v_{\lambda}^{\mathcal{B}}$. For simplicity of notation, from now on we omit the symbols with reference to $\lambda$.

(ii) For all $m \in \mathbb{N}$, denote the game representation of the $m$-repeated $\mathcal{B}$-associated game $\left\langle N, v^{m * \mathcal{B}}\right\rangle$ as a linear combination of the worth $v(T)$ for all coalitions $T \subseteq N, T \neq \emptyset$, by

$$
v^{m * \mathcal{B}}(S)=\sum_{\substack{T \subseteq N, T \neq \emptyset}} \gamma_{m, \mathcal{B}}^{S}(T) \cdot v(T) \text { for all } S \subseteq N, S \neq \emptyset
$$

We distinguish two tasks. The first task concerns the study of these game representations (8) in order to present an interrelationship between two game representations, switching from an arbitrary collection of constants $\mathcal{B}$ to the standard collection of unit constants $\mathcal{U}$. The forthcoming interrelationship (9) is rather appealing since it takes into account only the quotient of two constants from the collection $\mathcal{B}$. As an adjunct, we derive the extended version of (7) in that the $\mathcal{B}$-scaled game of the $m$ repeated $\mathcal{B}$-associated game equals the $m$-repeated standard associated game of the $\mathcal{B}$-scaled game, that is $\mathcal{B}\left(v^{m * \mathcal{B}}\right)=(\mathcal{B} v)^{m * \mathcal{U}}$. The two proofs are independent from Hamiache's work in the setting of the Shapley value. The second task concerns the study of convergence of any sequence $\left\{\gamma_{m, \mathcal{B}}^{S}(T)\right\}_{m=1}^{\infty}$, for fixed coalitions $S, T$. Due to the completion of the first task, the former sequence converges if and only if the standard sequence $\left\{\gamma_{m, \mathcal{U}}^{S}(T)\right\}_{m=1}^{\infty}$ converges, which convergence problems has been completely solved in Hamiache's work 2001. Nevertheless, since the outcomes of these convergent sequences are slightly different, it is necessary to present the last stage of a slightly adapted proof of uniqueness.

\subsection{Interrelationships between game representations}

Proposition 2 Concerning the n-person game representations (8), for all $m \in \mathbb{N}$, all $S \subseteq N, S \neq \emptyset$, for all $T \subseteq N, T \neq \emptyset$,

$$
\gamma_{m, \mathcal{B}}^{S}(T)=\frac{b_{t}^{n}}{b_{s}^{n}} \cdot \gamma_{m, \mathcal{U}}^{S}(T)
$$

Hence, $\left\langle N, \mathcal{B}\left(v^{m * \mathcal{B}}\right)\right\rangle=\left\langle N,(\mathcal{B} v)^{m * \mathcal{U}}\right\rangle$, i.e., $\left(\mathcal{B}\left(v^{m * \mathcal{B}}\right)\right)(S)=(\mathcal{B} v)^{m * \mathcal{U}}(S)$ for all $S \subseteq N$

Proof Let $\langle N, v\rangle \in \mathcal{G}$. The proof of (9) is rather technical (proceeding by induction on $m \geq 1$ ) and will be postponed till the appendix. However, (9) as well as 
the game representation (8) are helpful to establish the proof of the latter game equality $\left\langle N, \mathcal{B}\left(v^{m * \mathcal{B}}\right)\right\rangle=\left\langle N,(\mathcal{B} v)^{m * \mathcal{U}}\right\rangle$. For all $m \in \mathbb{N}$, all $S \subseteq N, S \neq \emptyset$,

$$
\begin{aligned}
\left(\mathcal{B}\left(v^{m * \mathcal{B}}\right)\right)(S) & =b_{s}^{n} \cdot\left(v^{m * \mathcal{B}}(S)\right) \stackrel{(8)}{=} \sum_{\substack{T \subseteq N, T \neq \emptyset}} \gamma_{m, \mathcal{B}}^{S}(T) \cdot b_{s}^{n} \cdot v(T) \\
& \stackrel{(9)}{=} \sum_{\substack{T \subseteq N, T \neq \emptyset}} \gamma_{m, \mathcal{U}}^{S}(T) \cdot b_{t}^{n} \cdot v(T)=\sum_{\substack{T \subseteq N, T \neq \emptyset}} \gamma_{m, \mathcal{U}}^{S}(T) \cdot(\mathcal{B} v)(T) \\
& \stackrel{(8)}{=}(\mathcal{B} v)^{m * \mathcal{U}}(S)
\end{aligned}
$$

\subsection{Convergence results}

Hamiache's results 5 and $6(2001$, p. 285) state that, for all coalitions $S$ and $T$, the standard sequence $\left\{\gamma_{m, \mathcal{U}}^{S}(T)\right\}_{m=1}^{\infty}$ converges provided $0<\lambda<\frac{2}{n}$ (the proof of result 6 is rather complex). Hence, by (9), for all coalitions $S, T$, and all collections of positive constants $\mathcal{B}$, the induced sequence $\left\{\gamma_{m, \mathcal{B}}^{S}(T)\right\}_{m=1}^{\infty}$ converges too. The limit is denoted by $\tilde{\gamma}_{\mathcal{B}}^{S}(T)$. Define the limit game $\left\langle N, \tilde{v}_{\mathcal{B}}\right\rangle$ by

$$
\tilde{v}_{\mathcal{B}}(S)=\sum_{\substack{T \subseteq N, T \neq \emptyset}} \tilde{\gamma}_{\mathcal{B}}^{S}(T) \cdot v(T) \text { for all } S \subseteq N, S \neq \emptyset
$$

By (8), the sequence $\left\{\left\langle N, v^{m * \mathcal{B}}\right\rangle\right\}_{m=1}^{\infty}$ of repeated $\mathcal{B}$-associated games converges (pointwise) to the limit game $\left\langle N, \tilde{v}_{\mathcal{B}}\right\rangle$. For any value $\Phi$ satisfying continuity, the corresponding sequence $\left\{\Phi\left(N, v^{m * \mathcal{B}}\right)\right\}_{m=1}^{\infty}$ of values converges to the value $\Phi\left(N, \tilde{v}_{\mathcal{B}}\right)$. If, in addition, the value $\Phi$ satisfies $\mathcal{B}$-consistency, then $\Phi\left(N, v^{m * \mathcal{B}}\right)=\Phi(N, v)$ for all $m \in \mathbb{N}$. Hence, by convergence, $\Phi\left(N, \tilde{v}_{\mathcal{B}}\right)=\Phi(N, v)$. In the setting of the standard collection with unit constants $\mathcal{U}$, Hamiache's result 8 (2001, p. 288) states that the limit game $\left\langle N, \tilde{v}_{\mathcal{U}}\right\rangle$ is inessential. In our current framework with reference to an arbitrary collection of positive constants $\mathcal{B}$, we claim that the limit game $\left\langle N, \tilde{v}_{\mathcal{B}}\right\rangle$ is $\mathcal{B}$-inessential, that is the $\mathcal{B}$-scaled game $\left\langle N, \mathcal{B}\left(\tilde{v}_{\mathcal{B}}\right)\right\rangle$ is inessential. The proof of the fundamental claim will be based on the following convergence results taken from Hamiache's former work.

Theorem 3 (Hamiache 2001, Results 7, 6, 5, pp.287, 285, 285 respectively).

Let $0<\lambda<\frac{2}{n}$, and $S \subseteq N, S \neq \emptyset$, and $T \subseteq N, T \neq \emptyset$. Then

$$
\tilde{\gamma}_{\mathcal{U}}^{S}(T)=\sum_{j \in S} \tilde{\gamma}_{\mathcal{U}}^{\{j\}}(T)
$$

$$
\tilde{\gamma}_{\mathcal{U}}^{S}(T)=\frac{1}{n-t} \cdot \sum_{j \in T} \tilde{\gamma}_{\mathcal{U}}^{S}(T \backslash\{j\}) \quad \text { provided } 2 \leq t<n .
$$


(iii)

$$
\tilde{\gamma}_{\mathcal{U}}^{S}(\{j\})= \begin{cases}\frac{n-s}{n \cdot(n-1)}, & \text { if } j \in S, \\ \frac{-S}{n \cdot(n-1)}, & \text { if } j \notin S .\end{cases}
$$

Corollary 1 Let $0<\lambda<\frac{2}{n}$, and $S \subseteq N, S \neq \emptyset$, and $T \subseteq N, T \neq \emptyset$. Then

$$
\tilde{\gamma}_{\mathcal{B}}^{S}(T)=\frac{b_{t}^{n}}{b_{s}^{n}} \cdot \tilde{\gamma}_{\mathcal{U}}^{S}(T)
$$

(ii)

$$
\tilde{\gamma}_{\mathcal{B}}^{S}(T)=\frac{b_{1}^{n}}{b_{s}^{n}} \cdot \sum_{j \in S} \tilde{\gamma}_{\mathcal{B}}^{\{j\}}(T)
$$

(iii)

$$
\tilde{\gamma}_{\mathcal{B}}^{S}(T)=\frac{1}{n-t} \cdot \frac{b_{t}^{n}}{b_{t-1}^{n}} \cdot \sum_{j \in T} \tilde{\gamma}_{\mathcal{B}}^{S}(T \backslash\{j\}) \quad \text { provided } 2 \leq t<n .
$$

$$
\tilde{\gamma}_{\mathcal{B}}^{S}(\{j\})= \begin{cases}\frac{b_{1}^{n}}{b_{s}^{n}} \cdot \frac{n-s}{n \cdot(n-1)}, & \text { if } j \in S, \\ \frac{b_{1}^{n}}{b_{s}^{n}} \cdot \frac{-s}{n \cdot(n-1)}, & \text { if } j \notin S .\end{cases}
$$

Notice that (14) follows immediately from (9) by convergence and in turn, (15)-(17) are direct restatements of (11)-(13) of Theorem 3.

Theorem 4 Let $\langle N, v\rangle \in \mathcal{G}$ and $0<\lambda<\frac{2}{n}$. Then the following holds:

(i) The limit game $\left\langle N, \tilde{v}_{\mathcal{B}}\right\rangle$ is $\mathcal{B}$-inessential, that is $\left\langle N, \mathcal{B}\left(\tilde{v}_{\mathcal{B}}\right)\right\rangle$ is inessential.

(ii) For all $T \subseteq N$ satisfying $2 \leq t<n$

$$
\begin{gathered}
\tilde{\gamma}_{\mathcal{B}}^{\{i\}}(T)=\frac{1}{n-t} \cdot \frac{b_{t}^{n}}{b_{t-1}^{n}} \cdot \sum_{j \in T} \tilde{\gamma}_{\mathcal{B}}^{\{i\}}(T \backslash\{j\}) \text { for all } i \in N ; \\
\tilde{\gamma}_{\mathcal{B}}^{\{i\}}(T)= \begin{cases}\frac{b_{t}^{n}}{b_{1}^{n}} \cdot \frac{(t-1) ! \cdot(n-t) !}{n !}, & \text { if } i \in T, \\
\frac{-b_{t}^{n}}{b_{1}^{n}} \cdot \frac{t ! \cdot(n-1-t) !}{n !}, & \text { if } i \notin T . \\
\tilde{v}_{\mathcal{B}}(\{i\})=\frac{S h_{i}(N, \mathcal{B} v)}{b_{1}^{n}} \text { for all } i \in N .\end{cases}
\end{gathered}
$$

The technical proof of Theorem 4 is postponed till the appendix.

Proof of the uniqueness part of Theorem 2

Parts (i) and (iii) of Theorem 4 remain to complete the uniqueness part of the characterization of Theorem 2. Suppose a value $\Phi$ on the universal game space $\mathcal{G}$ possesses the $\mathcal{B}$-inessential game property, continuity, and $\mathcal{B}$-consistency with respect to the $\mathcal{B}$ associated game of (6). Let $\langle N, v\rangle \in \mathcal{G}$. As shown in the paragraph above Theorem 3 , the latter two axioms imply the equality $\Phi\left(N, \tilde{v}_{\mathcal{B}}\right)=\Phi(N, v)$. By Theorem 4(i), the 
limit game $\left\langle N, \tilde{v}_{\mathcal{B}}\right\rangle$ is $\mathcal{B}$-inessential and thus, from the $\mathcal{B}$-inessential game property for $\Phi$ we derive the following:

$$
\Phi_{i}(N, v)=\Phi_{i}\left(N, \tilde{v}_{\mathcal{B}}\right)=b_{1}^{n} \cdot \tilde{v}_{\mathcal{B}}(\{i\}) \stackrel{(20)}{=} \operatorname{Sh}_{i}(N, \mathcal{B} v) \quad \text { for all } i \in N
$$

Hence, $\Phi(N, v)=\operatorname{Sh}(N, \mathcal{B} v)=\psi(N, v)$ for all games $\langle N, v\rangle$, where $\psi$ is the efficient, symmetric, and linear value on $\mathcal{G}$ induced by $\mathcal{B}$ (see (5)). So, $\Phi=\psi$.

\section{Concluding remarks}

In order to apply Theorem 2 to some subclasses of efficient, symmetric, and linear values, we discuss the class of least square values, which, in turn, includes the additive efficient normalization of any semi-value.

Example 2 The solidarity value $\operatorname{Sol}(N, v)$ of a game $\langle N, v\rangle$ is defined by some expected average marginal contribution over all coalitions containing the player as follows (Nowak 1994):

$$
\operatorname{Sol}_{i}(N, v)=\sum_{\substack{S \subseteq N, n \\
i \in S}} \frac{1}{\left(\begin{array}{c}
n-1 \\
s-1
\end{array}\right)} \cdot \sum_{j \in S}\left[\frac{v(S)-v(S \backslash\{j\})}{s}\right] \quad \text { for all } i \in N .
$$

It is known that the solidarity value is an efficient, symmetric, and linear value and in fact, its corresponding collection of scaling constants $\mathcal{B}=\left\{b_{s}^{n}\right\}$ is given by $b_{s}^{n}=\frac{1}{s+1}$ for all $1 \leq s \leq n-1$ with $b_{n}^{n}=1$. By Theorem 2 , the solidarity value is the unique value $\Phi$ on $\mathcal{G}$ verifying the $\mathcal{B}$-inessential game property, continuity, and $\mathcal{B}$-consistency with respect to the $\mathcal{B}$-associated game $\left\langle N, v_{\lambda}^{\mathcal{B}}\right\rangle$ of $(6)$ defined by $v_{\lambda}^{\mathcal{B}}(\emptyset)=0$ and for all $S \subseteq N, S \neq \emptyset$,

$$
\frac{v_{\lambda}^{\mathcal{B}}(S)}{s+1}=\frac{v(S)}{s+1}+\lambda \cdot \sum_{j \in N \backslash S}\left[\frac{v(S \cup\{j\})}{s+2}-\frac{v(S)}{s+1}-\frac{v(\{j\})}{2}\right] .
$$

Example 3 Let $\mathcal{P}=\left\{p_{s}^{n} \mid n \in \mathbb{N} \backslash\{0\}, \quad s=1,2, \ldots, n\right\}$ be a (yet unspecified) collection of probability distributions satisfying the normalization condition $\sum_{s=1}^{n}\left(\begin{array}{l}n-1 \\ s-1\end{array}\right) \cdot p_{s}^{n}=1$ and the inverse Pascal triangle conditions $p_{s}^{n-1}=p_{s}^{n}+p_{s+1}^{n}$ for all $s \in\{1,2, \ldots, n-1\}$, all $n \geq 2$. For all games $\langle N, v\rangle \in \mathcal{G}$, let the corresponding semi-value $\operatorname{SE}^{\mathcal{P}}(N, v)$ and its additive efficient normalization $\operatorname{ESE}^{\mathcal{P}}(N, v)$ be defined as follows (Ruiz 1998, pp. 119-120): for all $i \in N$

$$
\begin{aligned}
S E_{i}^{\mathcal{P}}(N, v) & =\sum_{\substack{S \subseteq N \\
i \in S}} p_{s}^{n} \cdot[v(S)-v(S \backslash\{i\})] \text { and } \\
\operatorname{ESE}_{i}^{\mathcal{P}}(N, v) & =S E_{i}^{\mathcal{P}}(N, v)+\frac{1}{n} \cdot\left[v(N)-\sum_{j \in N} S E_{j}^{\mathcal{P}}(N, v)\right] .
\end{aligned}
$$


Clearly, the additive efficient normalization satisfies efficiency and inherits linearity and symmetry from its underlying semi-value. Consequently, the additive efficient normalization of any semi-value is an efficient, symmetric, and linear value and in fact, its corresponding collection of scaling constants $\mathcal{B}=\left\{b_{s}^{n}\right\}$ is given by $b_{s}^{n}=s \cdot\left(\begin{array}{c}n-1 \\ s\end{array}\right) \cdot p_{s}^{n-1}$ for all $1 \leq s \leq n-1$ with $b_{n}^{n}=1$ for all $n \geq 2$. By Theorem 2 , for any collection of positive probability distributions $\mathcal{P}$, the additive efficient normalization $E S E^{\mathcal{P}}$ of the underlying semi-value $S E^{\mathcal{P}}$ is the unique value $\Phi$ on $\mathcal{G}$ verifying the $\mathcal{B}$-inessential game property, continuity, and $\mathcal{B}$-consistency with respect to the $\mathcal{B}$-associated game $\left\langle N, v_{\lambda}^{\mathcal{B}}\right\rangle$ of (6). Clearly, the Shapley value arises as the (unique efficient) semi-value $S E^{\mathcal{P}}$ where $p_{s}^{n}=\frac{1}{n \cdot\left(\begin{array}{c}n-1 \\ s-1\end{array}\right)}$ for all $1 \leq s \leq n$.

Example 4 Let $\langle N, v\rangle \in \mathcal{G}$. With every (yet unspecified) collection of nonnegative constants $\mathcal{M}=\left\{m_{s}^{n} \mid n \in \mathbb{N} \backslash\{0,1\}, s=1,2, \ldots, n-1\right\}$, there is associated the least square value $\operatorname{LS}^{\mathcal{M}}(N, v)$ to be defined as the unique optimal solution of the following optimization problem of which the objective function is given by the weighted sum of the square of the "excesses" of all non-trivial coalitions (the so-called least square problem, Ruiz 1998, pp.113-114):

$$
\begin{aligned}
& \text { Minimize } \sum_{\substack{S \subseteq N, S \neq N, S \neq \emptyset}} m_{s}^{n} \cdot\left[v(S)-\sum_{j \in S} x_{j}\right]^{2} \\
& \text { subject to } \mathbf{x}=\left(x_{j}\right)_{j \in N} \in \mathbb{R}^{N} \text { such that } \sum_{j \in N} x_{j}=v(N) .
\end{aligned}
$$

Ruiz et al. (1998, Theorem 8, p. 116) showed that the class of least square values is fully characterized by the following five properties: efficiency, linearity, symmetry, coalitional monotonicity, and inessential game property. As such, the least square family contains the additive efficient normalization of any semi-value. Particularly, it turns out that the Shapley value agrees with the least square value $L S^{\mathcal{M}}$ whenever $m_{s}^{n}=\frac{1}{(n-1) \cdot\left(\begin{array}{c}n-2 \\ s-1\end{array}\right)}$ for all $1 \leq s \leq n-1$. The solidarity value does not agree with any least square value since it violates the inessential game property.

In fact (Ruiz 1998, p. 114), every least square value $L S^{\mathcal{M}}$ is of the form (3) associated with the constants $\rho_{s}^{n}=\frac{s \cdot(n-s)}{n} \cdot \frac{m_{s}^{n}}{S_{\mathcal{M}}(n)}$ or equivalently, of the form (4) associated with the scaling constants $b_{s}^{n}=s \cdot\left(\begin{array}{c}n-1 \\ s\end{array}\right) \cdot \frac{m_{s}^{n}}{S_{\mathcal{M}}(n)}$ for all $1 \leq s \leq n-1$, where $S_{\mathcal{M}}(n):=\sum_{s=1}^{n-1}\left(\begin{array}{c}n-2 \\ s-1\end{array}\right) \cdot m_{s}^{n}$ for all $n \geq 2$.

By Theorem 2, for any collection of positive constants $\mathcal{M}$, the corresponding least square value $L S^{\mathcal{M}}$ is the unique value $\Phi$ on $\mathcal{G}$ verifying the $\mathcal{B}$-inessential game property, continuity, and $\mathcal{B}$-consistency with respect to the $\mathcal{B}$-associated game $\left\langle N, v_{\lambda}^{\mathcal{B}}\right\rangle$ of (6). 
Open Access This article is distributed under the terms of the Creative Commons Attribution Noncommercial License which permits any noncommercial use, distribution, and reproduction in any medium, provided the original author(s) and source are credited.

\section{Appendix: Two additional proofs}

Proof of Proposition 2. Let $\langle N, v\rangle \in \mathcal{G}$. The proof of (9) proceeds by induction on $m \geq 1$. If $m=1$, then it follows from (7) and the game representation (8) applied to the $\mathcal{B}$-scaled game, that it holds for all $S \subseteq N, S \neq \emptyset$,

$$
\begin{aligned}
b_{s}^{n} \cdot v^{\mathcal{B}}(S) & =\left(\mathcal{B}\left(v^{\mathcal{B}}\right)\right)(S) \stackrel{(7)}{=}(\mathcal{B} v)^{\mathcal{U}}(S) \\
& \stackrel{(8)}{=} \sum_{\substack{T \subseteq N, T \neq \emptyset}} \gamma_{1, \mathcal{U}}^{S}(T) \cdot(\mathcal{B} v)(T)=\sum_{\substack{T \subseteq N, T \neq \emptyset}} \gamma_{1, \mathcal{U}}^{S}(T) \cdot b_{t}^{n} \cdot v(T) .
\end{aligned}
$$

Thus, $v^{\mathcal{B}}(S)=\sum_{\substack{T \subseteq \emptyset \\ b_{s}^{n}}} \frac{b_{t}^{n}}{b_{1, \mathcal{U}}^{n}} \cdot \gamma_{1}^{S}(T) \cdot v(T)$ and hence, $\gamma_{1, \mathcal{B}}^{S}(T)=\frac{b_{t}^{n}}{b_{s}^{n}} \cdot \gamma_{1, \mathcal{U}}^{S}(T)$ for all $T \subseteq N, T \neq \emptyset$, due to the unique representation (8) applied to the term $v^{\mathcal{B}}(S)$. So, (9) holds if $m=1$.

Suppose (9) holds for $m$, that is $\gamma_{m, \mathcal{B}}^{S}(T)=\frac{b_{t}^{n}}{b_{s}^{n}} \cdot \gamma_{m, \mathcal{U}}^{S}(T)$ for all $S \subseteq N, S \neq \emptyset$, for all $T \subseteq N, T \neq \emptyset$. We aim to prove (9) for $m+1$.

Let $S \subseteq N, S \neq \emptyset$. We prove $\gamma_{m+1, \mathcal{B}}^{S}(T)=\frac{b_{t}^{n}}{b_{s}^{n}} \cdot \gamma_{m+1, \mathcal{U}}^{S}(T)$ for all $T \subseteq N, T \neq \emptyset$. For the $(m+1)$-repeated $\mathcal{B}$-associated game $\left\langle N, v^{(m+1) * \mathcal{B}}\right\rangle$, we have

$$
\begin{aligned}
& \left(v^{(m+1) * \mathcal{B}}\right)(S) \\
= & \left(v^{\mathcal{B}}\right)^{m * \mathcal{B}}(S) \quad \text { by using }(m+1) \text {-repeated } \mathcal{B} \text {-associated game } \\
\stackrel{(8)}{=} & \sum_{T \subseteq N,} \gamma_{m, \mathcal{B}}^{S}(T) \cdot v^{\mathcal{B}}(T) \quad \text { by }(8) \text { applied to the } \mathcal{B} \text {-associated game } \\
= & \sum_{T \neq \emptyset} \frac{b_{t}^{n}}{b_{s}^{n}} \cdot \gamma_{m, \mathcal{U}}^{S}(T) \cdot v^{\mathcal{B}}(T) \quad \text { by the induction hypothesis } \\
= & \sum_{T \neq \emptyset} \frac{\gamma_{m, \mathcal{U}}^{S}(T)}{b_{s}^{n}} \cdot\left(\mathcal{B}\left(v^{\mathcal{B}}\right)\right)(T) \quad \text { by using the } \mathcal{B} \text {-scaled game } \\
& \stackrel{(7)}{=} \sum_{T \subseteq N,} \frac{\gamma_{m, \mathcal{U}}^{S}(T)}{b_{s}^{n}} \cdot(\mathcal{B} v)^{\mathcal{U}}(T) \quad \text { by (7) } \\
& \stackrel{(8)}{=} \frac{1}{b_{s}^{n}} \cdot\left((\mathcal{B} v)^{\mathcal{U}}\right)^{m * \mathcal{U}}(S) \quad \text { by (8) applied to the associated game }
\end{aligned}
$$




$$
\begin{aligned}
& =\frac{1}{b_{s}^{n}} \cdot(\mathcal{B} v)^{(m+1) * \mathcal{U}}(S) \quad \text { by using }(m+1) \text {-repeated } \mathcal{U} \text {-associated game } \\
& \stackrel{(8)}{=} \frac{1}{b_{s}^{n}} \cdot \sum_{\substack{T \subseteq N, T \neq \emptyset}} \gamma_{m+1, \mathcal{U}}^{S}(T) \cdot(\mathcal{B} v)(T) \quad \text { by }(8) \text { applied to the game }\langle N, \mathcal{B} v\rangle \\
& =\sum_{\substack{T \subseteq N \\
T \neq \emptyset}} \gamma_{m+1, \mathcal{U}}^{S}(T) \cdot \frac{b_{t}^{n}}{b_{s}^{n}} \cdot v(T) \quad \text { by using the } \mathcal{B} \text {-scaled game. }
\end{aligned}
$$

Hence, $\gamma_{m+1, \mathcal{B}}^{S}(T)=\gamma_{m+1, \mathcal{U}}^{S}(T) \cdot \frac{b_{t}^{n}}{b_{s}^{n}}$ for all $T \subseteq N, T \neq \emptyset$, due to the unique representation (8) applied to the term $\left(v^{(m+1) * \mathcal{B}}\right)(S)$. This completes the inductive proof of (9). The proof of the equality $\left\langle N, \mathcal{B}\left(v^{m * \mathcal{B}}\right)\right\rangle=\left\langle N,(\mathcal{B} v)^{m * \mathcal{U}}\right\rangle$ has been completed before.

Proof of Theorem 4 (i) Let $S \subseteq N, S \neq \emptyset$. From the game representation (10) for the limit game $\left\langle N, \tilde{v}_{\mathcal{B}}\right\rangle$, together with (15), we derive the following:

$$
\begin{aligned}
\tilde{v}_{\mathcal{B}}(S) \stackrel{(10)}{=} \sum_{\substack{T \subseteq N, T \neq \emptyset}} \tilde{\gamma}_{\mathcal{B}}^{S}(T) \cdot v(T) \stackrel{(15)}{=} \sum_{\substack{T \subseteq N, T \neq \emptyset}}\left[\frac{b_{1}^{n}}{b_{s}^{n}} \cdot \sum_{j \in S} \tilde{\gamma}_{\mathcal{B}}^{\{j\}}(T)\right] \cdot v(T) \\
=\sum_{j \in S} \frac{b_{1}^{n}}{b_{s}^{n}} \cdot\left[\sum_{\substack{T \subseteq N \\
T \neq \emptyset}} \tilde{\gamma}_{\mathcal{B}}^{\{j\}}(T) \cdot v(T)\right] \stackrel{(10)}{=} \sum_{j \in S} \frac{b_{1}^{n}}{b_{s}^{n}} \cdot \tilde{v}_{\mathcal{B}}(\{j\})
\end{aligned}
$$

and hence,

$$
\left(\mathcal{B}\left(\tilde{v}_{\mathcal{B}}\right)\right)(S)=b_{s}^{n} \cdot \tilde{v}_{\mathcal{B}}(S)=\sum_{j \in S} b_{1}^{n} \cdot \tilde{v}_{\mathcal{B}}(\{j\})=\sum_{j \in S}\left(\mathcal{B}\left(\tilde{v}_{\mathcal{B}}\right)\right)(\{j\})
$$

This proves that the $\mathcal{B}$-scaled game $\left\langle N, \mathcal{B}\left(\tilde{v}_{\mathcal{B}}\right)\right\rangle$ is inessential, that is, the limit game $\left\langle N, \tilde{v}_{\mathcal{B}}\right\rangle$ itself is $\mathcal{B}$-inessential.

(ii) (18) is equivalent to (16) applied to a one-person coalition. Given (18), the proof of (19) proceeds by induction on $t \geq 1$. If $t=1$, then (19) reduces to $\tilde{\gamma}_{\mathcal{B}}^{\{i\}}(\{i\})=\frac{1}{n}$, and $\tilde{\gamma}_{\mathcal{B}}^{\{i\}}(\{j\})=\frac{-1}{n \cdot(n-1)}$ for all $j \in N \backslash\{i\}$, which equalities hold because of (17). So, (19) holds if $t=1$. Suppose (19) holds for coalitions of size $t-1$. We aim to prove (19) for any coalition $T$ of size $t, t \geq 2$. If $i \in T$, 


$$
\begin{aligned}
\tilde{\gamma}_{\mathcal{B}}^{\{i\}}(T) & \stackrel{(18)}{=} \frac{1}{n-t} \cdot \frac{b_{t}^{n}}{b_{t-1}^{n}} \cdot \sum_{j \in T} \tilde{\gamma}_{\mathcal{B}}^{\{i\}}(T \backslash\{j\}) \\
& =\frac{1}{n-t} \cdot \frac{b_{t}^{n}}{b_{t-1}^{n}} \cdot \frac{b_{t-1}^{n}}{b_{1}^{n}} \cdot\left[\frac{(t-1) ! \cdot(n-t+1) !}{n !}-\frac{(t-1) ! \cdot(n-t) !}{n !}\right] \\
& =\frac{1}{n-t} \cdot \frac{b_{t}^{n}}{b_{1}^{n}} \cdot \frac{(t-1) ! \cdot(n-t) !}{n !} \cdot(n-t) \\
& =\frac{b_{t}^{n}}{b_{1}^{n}} \cdot \frac{(t-1) ! \cdot(n-t) !}{n !}
\end{aligned}
$$

Thus, (19) is valid if $i \in T$. The case $i \notin T$ is left to the reader. This completes the inductive proof of (19). In fact, (19) yields for all $i \in N$, and all $S \subseteq N \backslash\{i\}$,

$$
\begin{aligned}
\tilde{\gamma}_{\mathcal{B}}^{\{i\}}(S) & =\frac{-b_{s}^{n}}{b_{1}^{n}} \cdot \frac{s ! \cdot(n-1-s) !}{n !} \quad \text { as well as } \\
\tilde{\gamma}_{\mathcal{B}}^{\{i\}}(S \cup\{i\}) & =\frac{b_{s+1}^{n}}{b_{1}^{n}} \cdot \frac{s ! \cdot(n-1-s) !}{n !}
\end{aligned}
$$

From this, together with the game representation (10) for the limit game $\left\langle N, \tilde{v}_{\mathcal{B}}\right\rangle$ applied to one-person coalitions, we derive for all $i \in N$

$$
\begin{aligned}
\tilde{v}_{\mathcal{B}}(\{i\}) & \stackrel{(10)}{=} \sum_{\substack{T \subseteq N \\
T \ni i}} \tilde{\gamma}_{\mathcal{B}}^{\{i\}}(T) \cdot v(T)+\sum_{T \subseteq N \backslash\{i\}, T \neq \emptyset} \tilde{\gamma}_{\mathcal{B}}^{\{i\}}(T) \cdot v(T) \\
& =\sum_{S \subseteq N \backslash\{i\}} \tilde{\gamma}_{\mathcal{B}}^{\{i\}}(S \cup\{i\}) \cdot v(S \cup\{i\})+\sum_{S \subseteq N \backslash\{i\}} \tilde{\gamma}_{\mathcal{B}}^{\{i\}}(S) \cdot v(S) \\
& \stackrel{(21)}{=} \frac{1}{b_{1}^{n}} \cdot \sum_{S \subseteq N \backslash\{i\}} \frac{s ! \cdot(n-1-s) !}{n !} \cdot\left[b_{s+1}^{n} \cdot v(S \cup\{i\})-b_{s}^{n} \cdot v(S)\right] \\
& =\frac{1}{b_{1}^{n}} \cdot \sum_{S \subseteq N \backslash\{i\}} \frac{s ! \cdot(n-1-s) !}{n !} \cdot[(\mathcal{B} v)(S \cup\{i\})-(\mathcal{B} v)(S)] \\
& \stackrel{(1)}{=} \frac{1}{b_{1}^{n}} \cdot S h_{i}(N, \mathcal{B} v)
\end{aligned}
$$

This completes the proof of (20).

\section{References}

Driessen TSH (1988) Cooperative games, solutions, and applications. Kluwer Academic Publishers, Dordrecht

Hamiache G (2001) Associated consistency and Shapley value. Int J Game Theory 30:279-289

Nowak AS, Radzik T (1994) A solidarity value for $n$-person transferable utility games. Int J Game Theory 23:43-48

Roth AE (ed) (1988) The Shapley value: essays in honor of Lloyd S. Shapley. Cambridge University Press, Cambridge 
Ruiz LM, Valenciano F, Zarzuelo JM (1998) The family of least square values for transferable utility games. Games Econ Behav 24:109-130

Shapley LS (1953) A value for $n$-person games. Ann Math Study 28:307-317. Also in Roth AE (1988), pp $31-40$ 